\title{
O papel das armadilhas com semioquímicos no manejo da broca-do- café, Hypothenemus hampei
}

\section{The role of semiochemical traps in the management of coffee berry borer Hypothenemus hampei}

\author{
Flávia Cloclet da Silva ${ }^{1}$; Maurício Ursi Ventura ${ }^{2 *}$, Lauro Morales ${ }^{3}$
}

Resumo

\begin{abstract}
A broca-do-café é a praga mais importante do cafeeiro. Provoca prejuízos em quantidade e qualidade da produção. Normalmente, é controlada por aplicações de inseticidas. Estratégias alternativas de manejo são necessárias principalmente pelo aumento da produção e demanda por produtos orgânicos. O uso de armadilhas para manejo da broca é revisado. A cor, o modelo da armadilha, os atraentes e as taxas de liberação são fatores diretamente relacionados com a eficiência das armadilhas. Metanol e etanol são os compostos utilizados como atraentes. Ocorrem interações entre estes fatores na atração da broca. As condições ambientais nas quais o cafeeiro é cultivado também devem influenciar as capturas. Estes aspectos podem explicar algumas diferenças em resultados de alguns estudos. Resultados conflitantes também são verificados no que se refere à eficiência das armadilhas quando utilizadas como estratégia única de controle no campo. Pesquisas adicionais devem ser incentivadas para entender com mais profundidade as interações entre a broca e a planta de café. Da mesma forma, deve-se estudar as interações dos vários fatores intrínsecos das armadilhas e destes fatores com o ambiente no qual a armadilha será instalada. $\mathrm{O}$ uso das armadilhas, associadas a outras estratégias de redução populacional da praga, é prática recomendável para manejo da broca.
\end{abstract}

Palavras chave: Semioquímicos, manejo, atraentes, armadilhas.

\begin{abstract}
The Coffee berry borer (CBB) is the most important pest in the coffee plantings. Losses are due to quality and quantity reductions. In most plantings, $\mathrm{CBB}$ is controlled using insecticides. Alternative management strategies are strongly demanded due to increasing production and demand for organic products. This paper revises the use of traps for $\mathrm{CBB}$ management. Color and trap designs, attractants and release rates are the factors directly related to trap efficiency. Methanol and ethanol mixture is used as attractant. Interactions among these factors in the CBB attraction are reported. Environment conditions under which the coffee plantings are conducted influence trap captures. These aspects may explain some differences in results for different studies. Conflicting results are also found in relation to traps efficiency when they are used as a unique control strategy in the field. Additional research may be encouraged to understand more deeply the interactions between the CBB and coffee plant. In the same way, we must study the interactions of the several intrinsic factors of the traps and the environment. The use of the trap, associated with other strategies to reduce pest population, is a recommended strategy for CBB management.
\end{abstract}

Key words: Semiochemicals, management, attractants, traps.

\footnotetext{
Bióloga, mestre em Agronomia na Universidade Estadual de Londrina

2 Professor do Depto. de Agronomia, Universidade Estadual de Londrina, CCA, , Londrina, PR, Brasil.

Engenheiro Agrônomo doutor em Entomologia, EMATER, PR.

* Autor para correspondência
} 


\section{Introdução}

A broca-do-café, Hypothenemus hampei, é a principal praga do café em vários países produtores (DAMON, 2000). É originária da África Equatorial e foi descrita por Ferrari em 1867 (BENNASSI, 1989). A partir desta data, vários registros do aparecimento da praga ocorreram em diversos países da África, Europa e América Latina (PASCUAL et al., 2002). No Brasil, a broca-do-café foi referida em 1922 (PARRA; BATISTA; ZUCCHI, 1992), entretanto, acredita-se que sua introdução ocorreu por volta de 1913, em amostras vindas do Congo Belga para a região de Campinas (BENASSI, 1989).

Populações da praga podem causar perdas de até $80 \%$ da produção (REIS, 2002). Por passar grande parte do ciclo de vida no interior dos frutos do café, a broca é inseto de difícil controle. Somente quando as fêmeas abandonam os frutos para colonizarem outros, é que se tornam expostas aos com produtos químicos (MORALLO-REJEUS; BALDOS, 1980).

H. hampei é uma praga presente em grãos verdes e maduros de café, não causando danos às folhas, caules e ramos (DAMON, 2000; REIS, 2002). As fêmeas adultas abrem galerias no endosperma da semente, onde depositam os ovos. As larvas e os adultos alimentam-se das sementes provocando redução no peso dos grãos, que pode variar de 5 a $20 \%$, depois de processado (GUTIÉRREZMARTINEZ; RIVAS; SÁNCHES, 1993a; BORBÓN-MARTINEZ; MORA- ALFARO; GONZALES, 2000). Além disso, as lesões causadas nos frutos de café propiciam a entrada de fungos e bactérias, agentes responsáveis pelo apodrecimento e queda dos frutos, o que prejudica consideravelmente a qualidade da bebida (SPONAGEL, 1994).

Em muitos países, inclusive no Brasil, o inseticida organoclorado endosulfan, tem sido adotado como o principal estratégia de controle de H. hampei (WATERHOUSE; NORRIS, 1989; SPONAGEL, 1994). Entretanto, populações da broca-do-café resistentes ao endosulfan foram constatadas na Nova Caledônia (BRUN et al., 1989; BRUN;
MARCILLAUD; GAUDICHON, 1994). Guzman (apud DAMON, 2000), observou mortalidade acentuada de parasitóides de $H$. hampei após as aplicações de endosulfan. Os inseticidas químicos não são permitidos em cultivos orgânicos. $\mathrm{O}$ cultivo orgânico de café, embora represente apenas 0,02\% da produção brasileira (MOREIRA, 2003), é uma atividade crescente no País. O Brasil é o maior produtor mundial de café convencional e sexto de café orgânico, mas acredita-se que o país tenha condições de alcançar o primeiro lugar na produção mundial de café orgânico (MOREIRA, 2003). Por estas razões, o controle alternativo da broca é uma necessidade imediata. Neste artigo, revisa-se e discute-se o uso de armadilhas no manejo da brocado-café.

\section{Estímulos visuais}

Ainda não é completo o entendimento dos fatores que influenciam a atração da broca-do-café e o seu comportamento durante o processo de colonização do hospedeiro. A distância entre o inseto e o hospedeiro foi descrita como o fator determinante para a determinação de qual estímulo é o mais significativo (ALONZO, 1984). Características ópticas parecem ser também imprescindíveis na orientação da broca-do-café (TICHELER, 1963).

No período em que as brocas saem dos frutos de café, são ditas fêmeas colonizadoras. Nesta fase, são atraídas por compostos voláteis dos frutos, etanol (E) e metanol (M), porém quando ainda não saíram dos frutos, estas fêmeas não apresentam respostas a estes semioquímicos (MATHIEU et al., 1991). Já em relação às respostas a estímulos visuais, todas as fêmeas mostram-se responsivas. A umidade (BAKER; BARRERA; RIVAS, 1992) e a luz (MATHIEU; BRUN; FRÉROT, 1997a) são fatores determinantes da saída das brocas aptas para oviposição dos frutos. Reportam-se capturas em grandes quantidades da broca-do-café após a ocorrência de precipitações nos cafezais (MATHIEU et al., 1999). 
Alguns estudos sugerem que fêmeas adultas de $H$. hampei são capazes de distinguir e selecionar os frutos de café de acordo com sua coloração, mostrando preferência significativa para frutos maduros (vermelhos) em comparação a frutos imaturos (verdes). Tal preferência foi demonstrada em estudos em laboratório e no campo (MENDONZA-MORA, 1991; GIORDANENGO; BRUN; FRÉROT, 1993; MATHIEU et al., 1997b; MATHIEU et al., 1991). Todavia, os resultados de estudos envolvendo a seleção de cores por fêmeas da broca-do-café são conflitantes, reforçando a hipótese de que vários fatores, além da cor, estão envolvidos no processo de localização e colonização de frutos de café por fêmeas de $H$. hampei (MENDONZA-MORA, 1991; MATHIEU; BRUN; FRÉROT, 1997a).

Maiores capturas de broca-do-café por armadilhas vermelhas em comparação às brancas (armadilhas modelo "hampei”), foram verificadas (MATHIEU et al., 1997b). Entretanto, BorbónMartinez, Mora-Alfaro e Gonzales (2000), registraram capturas 7,5 vezes maiores em armadilhas brancas do que em vermelhas. Capturas similares da broca-do-café foram obtidas quando se compararam armadilhas de garrafas transparentes, verde-transparentes e vermelhas (SILVA; VENTURA; MORALLES, 2005). Além das cores, o fundo que determina o contraste com a cor da armadilha é fator importante na atratividade da broca (MATHIEU et al., 1997b).

\section{Estímulos olfativos}

Substâncias emanadas pela planta de café atraem a broca-do-café (GIORDANENGO; BRUN; FRÉROT, 1993). O estudo destes semioquímicos tem possibilitado a descoberta de voláteis para utilização no manejo da broca (NORDLUND; LEWIS, 1976).

A atração da broca-do-café por extratos de fruto de café foi primeiramente relatada por Prates (1969). Neste estudo, foram utilizados extratos aquosos de frutos verdes e maduros em diferentes concentrações, observando-se maior resposta das fêmeas por extratos puros ou em concentrações acima de 50\%. Segundo Gutierrez-Martinéz, Rivas e Sánches (1993a), o grau de ataque dos frutos de café pode variar de acordo com diferentes fatores como a espécie e variedade da planta, fenologia dos frutos, localização geográfica e principalmente, a concentração de semioquímicos voláteis produzidos pela planta. Mendonza-Mora (1991) relataram a atração das fêmeas pelo E e M e pela mistura dos dois compostos.

Em estudos para determinar os voláteis dos frutos de café, 31 compostos foram detectados, embora apenas 11 tenham sido caracterizados (LIMA et al., 2004). Estes compostos puros não foram atrativos a fêmeas da broca-do-café. O benzaldeído, salicilato de metila e etila e 2-heptanona $(0,5$ ou 1,0\%) foram atrativos. A adição do benzaldeído ou salicilato de metila à mistura $\mathrm{E}: \mathrm{M}$ aumentou as capturas das armadilhas no campo.

Relatos de que as fêmeas da broca-do-café são atraídas por armadilhas iscadas com extratos de frutos de café e cafeína, além do M e E, que atuam como cairomônios na comunicação química entre o inseto e a planta hospedeira (GUTIÉRREZMARTINEZ; RIVAS; SÁNCHES, 1993b) indicaram a necessidade de maior investigação nesta área. Por outro lado, vários estudos confirmaram a atratividade do E e M para fêmeas da broca-do-café, reforçando seu potencial como isca para captura deste inseto em armadilhas (GUTIERREZ-MARTINEZ; ONDARZA, 1996; MATHIEU et al., 1997b; CÁRDENAS, 2000; VILLACORTA et al. 2001; OKUMURA et al., 2003). A questão da importância da cafeína na atração da broca e controvertida. Enquanto Gutierrez-Martinez, Rivas e Sánches (1993b), relataram sua importância na captura da broca, outros autores questionam como que um composto de baixa volatilidade poderia atrair os insetos, principalmente a maiores distância (ROJAS, 2005). 


\section{Estímulos visuais e olfativos}

A utilização de armadilhas para captura de escolitídeos é uma prática que tem sido adotada para o monitoramento e controle de importantes pragas de essência florestais (MOECK, 1970; CARRANO; MARQUES; PEDROSA, 1987). Para captura da broca-do-café, vários modelos de armadilhas já foram propostos. Dentre eles, se destacam o modelo de funil múltiplo (LINDGREN, 1983); armadilha ESALQ/84 (BERTI FILHO; FLECHTMANN, 1986); a armadilha "hampei" desenvolvida pelo CENICAFE na Colômbia e mais recentemente, o modelo feito com garrafas de plástico descartável de 2 L (modelo IAPAR) (VILLACORTA et al., 2001).

Okumura et al. (2003), compararam diferentes alturas de fixação de armadilhas de garrafa plástica em terreiros de secagem de café e não observaram diferenças entre as capturas de armadilhas fixadas a 0; 35 e $70 \mathrm{~cm}$ de altura do solo. Outros autores recomendam que as armadilhas sejam fixadas entre 1,0 e 1,5 m do solo e próximos às plantas de café (GUTIERREZ-MARTÍNEZ; RIVAS; SÁNCHES, 1993a; VILLACORTA et al. 2001). Quando alturas de fixação de 0,$5 ; 1,0$ e 1,5 foram comparadas no interior de lavouras não foram constatadas diferenças (SILVA; VENTURA; MORALLES, 2005). Barrera (2005), verificou que as capturas da broca-do-café em diferentes alturas variam de acordo com a fenologia da planta de café. No período de entressafra, tendem a voar em alturas superiores, enquanto que, no período reprodutivo são capturadas em alturas mais baixas.

O E e o M são referidos como atraentes da broca em vários trabalhos (BENASSI, 1990; MENDONZA-MORA, 1991; BORBÓNMARTÍNEZ; MORA-ALFARO; GONZALES, 2000; VILLACORTA et al., 2001). Os álcoois podem ser atrativos quando utilizados isoladamente. Porém, apresentam sinergismo quando usados combinados. Resultados similares nas capturas entre as misturas 1:1 e 1:3 foram reportadas, o que permite reduzir consideravelmente o custo do atraente, uma vez que o etanol custa cerca de $25 \%$ do metanol (SILVA; VENTURA; MORALLES, 2005).

As taxas nas quais os voláteis são liberados também influenciam significativamente as capturas. Comparação dos resultados de trabalhos mostram discrepância entre os resultados obtidos. Captura em armadilhas foram inicialmente referidas como decrescentes com o aumento da dose da mistura 1:3 (MENDONZA MORA, 1991; MATHIEU et al. 1997b). Entretanto, capturas crescentes com doses crescentes da mesma mistura foram relatadas na Nicarágua (BORBÓN-MARTINEZ; MORAALFARO; GONZALES, 2000). Resultados similares de capturas, utilizando diversas doses foram relatados por Silva, Ventura e Moralles. (2005), que utilizou a mistura 1:1. As diferenças nas concentrações das misturas, as condições do ambiente e diferenças nos modelos da armadilha podem ter determinado as diferenças nos resultados.

As cores podem interagir com os voláteis. Silva, Ventura e Moralles (2005), verificaram que armadilhas verde-transparentes com orifício de $2 \mathrm{~mm}$ no frasco liberador capturaram significativamente mais brocas adultas do que armadilhas verdes, com outros tamanhos de orifício e armadilhas vermelhas ou transparentes com várias taxas de liberação. Interações entre cores e taxas de liberação também foram reportadas por Mathieu et al. (1997b) e Lima et al. (2004).

\section{Papel no manejo}

As armadilhas podem ser utilizadas no manejo da broca-do-café para monitoramento ou controle em massa da praga. Uma relação linear entre as capturas e as infestações nos frutos foi obtida no campo (MATHIEU et al., 1999). Desta forma, verificou-se que as armadilhas podem ser utilizadas para mapear os locais, ou talhões, nas lavouras onde existem as maiores populações da broca, ou seja, caracterizase a infestação no espaço. Entretanto, ainda não se 
dispõe de estratégia de utilização das armadilhas para caracterizar o momento correto para estabelecer estratégias de controle, tais como pulverização de inseticidas ou fungos.

Para controle em massa, poucos estudos têm se preocupado em estabelecer relações entre as áreas com armadilhas instaladas no campo e a progressão das infestações nos frutos em comparação com áreas sem as armadilhas. Villacorta et al. (2001) reportaram a diminuição da intensidade de infestação da broca nos frutos para menos de 3\% em áreas com 25 armadilhas por ha, enquanto que nas áreas sem armadilhas foram constatadas taxas acima de $10 \%$. Neste caso, foram utilizadas garrafas plásticas de 2 L verde-transparentes. Entretanto, Moralles et al. (2005) testaram o uso de 30 e 35 armadilhas transparente por ha, mas não conseguiram manter as populações do inseto abaixo do nível de dano econômico, apesar de tê-las reduzido. Redução no número de frutos brocados, grãos quebrados e com outros defeitos e melhoria na qualidade da bebida, em estudo realizado durante 20 meses, foram relatados pela utilização de armadilha (LIMA et al. 2004). Entretanto, os autores não se referiram à manutenção dos danos abaixo do nível de controle. Além da utilização das armadilhas na lavoura, propõese também sua colocação nos terreiros de café, com o propósito de reduzir as populações que reinfestariam as lavouras numa safra seguinte (OKUMURA et al., 2005).

Uma outra questão que merece investigação é o uso de armadilhas no período de entressafra. Esta estratégia visaria substituir total ou parcialmente o repasse e varrição, práticas recomendadas para diminuição da população da broca pela retirada de frutos atacados. Estes frutos servem para o desenvolvimento das populações da broca que infestariam os frutos na próxima safra.

\section{Considerações finais}

Vários fatores estão envolvidos na determinação da eficiência das armadilhas. Fatores intrínsecos, como cores, atraentes, taxas de liberação, altura e local de fixação (planta ou estaca), contraste com a paisagem etc. interagem entre si e devem interagir com características do ambiente, p.e., o sistema de produção do café (adensado, semi-adensado e convencional), sombreado ou não, variedade, micro clima, relevo etc. A eficiência de armadilhas no controle da broca em café a pleno sol foi questionada (DUFOUR, 2004), e segundo o autor, esta estratégia é mais adequada para café sombreado. Desta forma, a contínua avaliação destes aspectos é condição básica para se conseguir estabelecer e incrementar consideravelmente a eficiência da armadilha. Este aumento de eficiência poderá reduzir os custos diretos (atraentes) e indiretos (trabalho) no uso da técnica, tornando sua relação custo benefício satisfatória.

Alguns produtores têm conseguido manter as infestações da broca-do-café abaixo do nível de controle pela utilização de armadilhas e colheita sanitária (VENTURA, 2005). Particularmente os produtores orgânicos, que não utilizam inseticidas químicos, têm nas armadilhas uma estratégia definitiva para reduzir as populações da broca. Obviamente, esta estratégia não deve ser considerada única, mas sim associada a outras práticas que reduzam a população da praga. É fundamental que a pesquisa avalie criteriosamente cada uma das estratégias.

Por outro lado, muitos produtores têm reclamado da eficiência do controle com inseticidas, provavelmente pela resistência em amostrar os frutos no campo, ou seja, a pulverização pode estar sendo feita no momento inadequado. Desta forma, a armadilha também apresenta potencial em estudos de monitoramento para definição da época correta de pulverização. 


\section{Referências}

ALONZO, F.R. Aspectos ecológicos de la broca Hypothenemus hampei (Scolytidae: Coleoptera). In.: El problema de la broca (Hypothenemus hampei Ferr.) (Col.: Scolytidae) y la caficultura: aspectos relacionados com importancia, daño, identificación, biología, ecología y control. San Jose, Costa Rica: IICA PROMECAFE, 1984, p. 71-136.

BAKER, P. S.; BARRERA, J.F.; RIVAS, A. Life-history studies of the coffee berry borer (Hypothenemus hampei, Scolytidae) on coffee trees in southern Mexico. The Journal of Applied Ecology, Oxford, v.29, n.3, p.656-662, 1992.

BARRERA, J. F. Investigación sobre la broca del café en México: logros, retos y perspectivas. In. BARRERA, J. R. Situatión actual e perspectivas de investigatión de manejo de la broca del café en Costa Rica, Cuba, Guatemala e México. Tapachula: Sociedade Mexicana de Entomologia, [19-]. p.14-21

BENASSI, V.L.R.M. A broca-do-café. Vitória.: EMCAPA, 1989.

BENASSI, V.L.R.M. Resultados preliminares da flutuação populacional da broca-do-café Hypothenemus hampei (Ferrari 1867) (Coleoptera: Scolytidae), na região norte do Espírito Santo. In CONGRESSO BRASILEIRO DE PESQUISAS CAFEEIRAS, 16., 1990, Espírito Santo do Pinhal. Anais... Rio de Janeiro: IBC, 1990. p.83.

BERTI FILHO, E.; FLECHTMANN, C. A. H. A model of ethanol trap to collect Scolytidae e Platypodidae (Insecta, Coleoptera). IPEF, Piracicaba, v.34, p.53-56, 1986.

BORBÓN MARTÍNEZ, O.; MORA-ALFARO, A.C.O.; GONZALES, L.M. Proyeto de trampas, atrayentes y repelentes para el control de la broca del fruto de cafeto, Hypothenemus hampei (Coleoptera: Scolytidae). In: SIMPOSIO LATINOAMERICANO DECAFEICULTURA, 19., 2000, San Jose (Costa Rica,). Anais... San José (Costa Rica): IICA-Promecafe, 2000. p.341-348.

BRUN, L. O.; MARCILLAUD, C.; GAUDICHON, V. Cross resistance between insecticides in coffee berry borer Hypothenemus hampei (Coleoptera: Scolytidae) from New Caledonia. Bulletin of Entomological Research, Farnham Royal, v.84, p.175-178, 1994.

BRUN, L. O., MARCILLAUD, C.; GAUDICHON, V.; SUCKLING, D.M. Endosulfan resistance in Hypothenemus hampei (Coleoptera: Scolytidae) in New Caledonia. Journal of Economic Entomology, Lanham, v.82, p.13111316,1989.

CÁRDENAS, R. M. Trampas y atrayentes para monitoreo de poblaciones de broca del café Hypothenemus hampei ( Ferrari) (Coleoptera: Scolytidae). In SIMPOSIO
LATINOAMERICANO DECAFICULTURA, 19., 2000, San Jose (Costa Rica). Anais...San Jose (Costa Rica): IICAPromecafe, 2000. p.369-379

CARRANO, A. F.; MARQUES, E. N.; PEDROSA, J. H. Análise comparativa entre dois modelos de armadilhas de impacto na coleta de Scolytidae. In: CONGRESSO BRASILEIRO DE ENTOMOLOGIA, 21., 1987, Campinas. Resumos... Campinas: Instituto Agronômico de Campinas, 1987.

CURE, J. R.; SANTOS, R. H. S.; MORAES, J. C.; VILELA, E. F.; GUTIERREZ, A. P. Fenologia e dinâmica populacional da broca-do-café Hypothenemus hampei (Ferr.) relacionadas às fases de desenvolvimento do fruto. Anais da Sociedade Entomologica do Brasil, Jaboticabal, v.27, n.3, p.325-335, 1998.

DAMON, A. A review of the biology and control of the coffee berry borer, Hypothenemus hampei (Coleoptera: Scolytidae). Bulletin Entomological Research, Farnham Royal, v.90, p.453-465, 2000.

DUFOUR, B. P. Condiciones de uso de las trampas en el control de la broca del café. In: WORKSHOP INTERNACIONAL SOBRE O MANEJODA BROCA-DOCAFÉ. 2004, Londrina. Resumos.... Londrina: UEL, 2004.

GIORDANENGO, P.; BRUN, L. O.; FREROT, B. Evidence for the allelochemical attraction of the coffee berry borer Hypothenemus hampei by coffee berries. Journal of Chemical Ecology, New York, v.19, n.4, p.763-769, Apr. 1993.

GUTIÉRREZ-MARTÍNEZ, A.; RIVAS, A. S. H.; SÁNCHES, A. V. Efecto de los diferentes extractos de cafe robusta Coffea canephora Piere ex Froehner sobre la captura de la broca del cafe Hypothenemus hampei Ferrari (Coleoptera: Scolytidae). In: SIMPOSIO DE CAFEICULTURA LATINOAMERICANA, 16., 1993. Managua (Nicarágua). Anais... Managua (Nicarágua): IICA/PROMECAFÉ, $1993 \mathrm{~b}$. v.2, p.1-7.

GUTIÉRREZ-MARTÍNEZ, A.; RIVAS, A. S. H.; SÁNCHES, A. V. Trampeo en el campo de la broca del fruto de cafe Hypothenemus hampei Ferrari (Coleoptera: Scolytidae) com los semioquimicos volatiles del fruto de cafe robusta Coffea canephora Piere ex Froehner. In: SIMPOSIO DE CAFEICULTURA LATINOAMERICANA, 16., 1993. Managua (Nicarágua). Anais... Managua (Nicarágua): IICA/PROMECAFÉ, 1993a. v.2, p.1-7.

GUTIÉRREZ-MARTÍNEZ, A.; ONDARZA, R. N. Kairomone effect of extracts from Coffea canephora over Hypothenemus hampei (Coleoptera: Scolytidae). Environmental Entomology, Madson, v.25, p.96-100, 1996.

(LIMA et al. 2004). 
LINDGREN, B. S. A multiple funnel trap for scolytid beetles (Coleoptera). Canadian Entomologist, Ottawa, v.115, n.3, p.299-302, 1983.

MATHIEU, F.; BRUN, L. O.; FREÂROT, B.; SUCKLING, D. M.; FRAMPTON, C. Progression in field infestation is linked with trapping of coffee berry borer, Hypothenemus hampei (Col., Scotytidae). Journal of Applied Entomology, Berlin, v.123, n.9, p.535-540, 1999.

MATHIEU, F.; BRUN, L. O.; FRÉROT, B. Factors related with native host abandon by the coffee berry borer. Journal of Applied Entomology, Berlin, v.121, n.3, p.175-180, 1997a.

MATHIEU, F.; BRUN, L.O.; MARCHILLAUD, C.; FRÉROT, B. Trapping of the coffee berry borer hypothenemus hampei Ferr. (Col., Scolytidae) within a mesh-enclosed environment: interaction of olfactory and visual stimuli. Journal of Applied Entomology, Berlin, v. 121, p.181-186, $1997 \mathrm{~b}$.

MATHIEU, F.; GAUDICHON, V.; BRUN, L. O.; FRÉROT, B. Effect of physiological status on olfactory and visual responses of female Hypothenemus hampei during host plant colonization. Physiological Entomology, Oxford, v.26, n.3, p.189-193, 1991.

MENDOZA MORA, J. R. Resposta da broca-do-café Hypothenemus hampei, a estímulos visuais $e$ semioquímicos. 1991. Tese (Doutorado) - Universidade Federal de Viçosa, Viçosa (MG).

MOECK, H. A. Ethanol as the primary attractant for the ambrosia beetle Trypodendron lineatum (Coleoptera: Scolytidae). Canadian Entomologist, Ottawa, v.102, n.8, p.985-995,1970.

Moralles et al. (2005não publicado)

MORALLO-REJESUS, B.; BALDOS, E. The biology of the coffee berry borer Hypothenemus hampei (Ferr.) (Scolytidae: Coleoptera) and its incidence in the southern Tagalog provinces. Philippine Entomologist, Manila (Filipinas), v.4, p.303-316, 1980.

MOREIRA, C. F. Caracterização de sistemas de produção de café orgânico sombreado e a pleno sol no sul de Minas Gerais. 2003. Dissertação (Mestrado) - Escola Superior de Agronomia Luiz de Queiroz da Universidade de São Paulo, Piracicaba.

NORDLUND, D. A; LEWIS, W.J. Terminology of chemicals releasing stimule in intraspecific and interspecific interactions. Journal of Chemical Ecology, New York, v.2, p.211-220, 1976.

OKUMURA, A. S. K.; NEVES, P.M.O.J.; POSSSAGNOLO, A. F.; CHOCOROSQUI, V. R.; SANTORO, P. H. Controle da broca-do-café Hypothenemus hampei (Ferrari) em terreiros de secagem de café. Semina, Londrina, v.24, n.2, p.277-282, 2003.
PARRA, J. R. P.; BATISTA, G. C.; ZUCCHI, R. A. Pragas do cafeeiro, p. 355-386. In: FEALQ (org.). Curso de Entomologia Aplicada a Agricultura. Piracicaba: FEALQ, 1992.

PASCUAL, H. V.; RUIZ, B. B.; CÁRDENAS, S. D.;LHÁVEN GÓMEZ, J. M.; VELÁZQUEZ, A. F. V. Respuesta de la broca del fruto Hypothenemus hampei Ferr.: a extractos e cerezas de café utilizados como atrayentes en Tepatlaxco. Veracruz: Sistema de Investigación del Golfo de México, 2002.

PRATES, H. S. Observações preliminares da atração da broca-do-café, Hypothenemus hampei (Ferrari, 1867), a extratos de frutos do cafeeiro (cereja e verde). $O$ solo, Piracicaba, v.61, p.13-14, 1969.

REIS, P. R. Broca-do-café: conheça os métodos para eliminar a ameaça. Cultivar, Pelotas, v.38, p.10-13, 2002.

ROJAS, J. Ecologia quimica de la broca del café y sus parasitoides. In. BARRERA, J. R. Situatión actual e perspectivas de investigatión de manejo de la broca del café en Costa Rica, Cuba, Guatemala e México. Tapachula: Sociedade Mexicana de Entomologia, 2005. p.14-21.

SILVA, F. C.; VENTURA, M. U.; MORALLES, L. Capture of coffee berry borers, Hypothenemus hampei (Coleoptera, Scolytidae), in response to trap characteristics. Scientia agricola, Piracicaba, 2005. (no prelo).

SPONAGEL, K.W. La broca del cafe Hypothenemus hampei en plantaciones de cafe robusta en la Amazonía Ecuatoriana. Giessen, Germany: Wissenschaftlicher, Fachverlag, 1994.

TICHELER, J. H. G. Estudio analítico de la epidemiología del escolitídeo de los granos de cafe Stephanoderes hampei, Ferr., en Costa del Marfil. Cenicafe, Chinchina, Colombia, v.14, n.4, p.223-294, 1963.

VENTURA, 2005), dados não publicados

VILLACORTA, A.; A. F. POSSAGNOLO, A. F. ; SILVA, R. Z.; RODRIGUES, P. S. Um modelo de armadilha com semioquímicos para o manejo integrado da broca do café Hypothenemus hampei (Ferrari) no Paraná.. In SIMPÓSIO BRASILEIRO DE PESQUISA DOS CAFÉS DO BRASIL, 2., 2001, Vitória. Anais... Brasília: Embrapa-Café, 2001. p.2093-2098

WATERHOUSE, D. F.; NORRIS, K. R. Biological control: pacific prospects. Canberra: Australian Centre for Internacional Agricultural Research, 1989. Sup.1. 
